\title{
International Society of Nephrology 0 by 25 Project: Lessons Learned
}

\author{
Etienne Macedo ${ }^{a}$ Guillermo Garcia-Garcia ${ }^{b}$ Ravindra L. Mehta ${ }^{a}$ \\ Michael V. Rocco ${ }^{c}$ \\ ${ }^{a}$ Department of Medicine, University of California San Diego, San Diego, CA, USA; ${ }^{b}$ Nephrology Service, Hospital \\ Civil de Guadalajara Fray Antonio Alcalde, University of Guadalajara Health Sciences Center, Guadalajara, Mexico; \\ 'Wake Forest School of Medicine, Medicine, Winston-Salem, NC, USA
}

\section{Keywords}

Acute kidney injury · Dehydration · Mortality · Epidemiology
J Am Soc Nephrol 2017]. In this review, we will comment on the main findings and lessons learned from the 0 by 25 initiative.

(c) 2019 The Author(s)

Published by S. Karger AG, Basel

\begin{abstract}
Acute kidney injury (AKI) is a common disorder with a high risk of mortality and development of chronic kidney disease. With the validation of the recent classification systems, RIFLE in 2004 and KDIGO, in use today, our understanding of AKI has evolved. We now know that community-acquired AKI is also associated with an increased risk of worse outcomes. In addition, several epidemiological studies, including cohorts from low-income and low-middle income countries, have confirmed common risk factors for communityacquired AKI. In 2013, the International Society of Nephrology launched the 0 by 25 campaign with the goal that no patient should die from preventable or untreated AKI in low-resource areas by 2025 [Mehta et al.: Lancet 2015; 385:2616-43]. The initial effort of the initiative was a metaanalysis of AKI epidemiology around the world. The second project of the 0 by 25 initiative, the Global AKI Snapshot (GSN) study, provided insights into the recognition, treatment, and outcomes of AKI worldwide [Mehta et al.: Lancet 2016;387:2017-25]. Following the GSN, a Pilot Project was designed to test whether education and a simple protocolbased approach can improve outcomes in patients at risk of community-acquired AKI in low-resource settings [Macedo:
\end{abstract}

\section{Introduction of 0 by 25 Projects}

The worldwide application of the RIFLE/AKIN (Risk, Injury, Failure, Loss of kidney function, and End-stage kidney disease/Acute Kidney Injury Network) and KDIGO (Kidney Disease: Improving Global Outcomes) classification systems has confirmed the increasing incidence of AKI in different settings [5-9]. The efforts of nephrology and critical care societies to create a unified classification system have enabled comparisons of AKI incidence and outcomes across diverse populations. The resultant epidemiological studies have shown increasing severity of AKI cases and a higher risk of death associated with AKI, in both hospital and community settings [10-12]. In addition, AKI is now a recognized important risk factor for new-onset chronic kidney disease (CKD), determining acceleration in the progression to end-stage renal disease [13-15].

The International Society of Nephrology (ISN) 0 by 25 initiative aims to eliminate or at least reduce avoidable AKI-related deaths around the world by 2025 [1]. Two key

\begin{tabular}{|c|c|}
\hline KARGER & $\begin{array}{l}\text { (C) } 2019 \text { The Author(s) } \\
\text { Published by S. Karger AG, Basel }\end{array}$ \\
\hline $\begin{array}{l}\text { E-Mail karger@karger.com } \\
\text { www.karger.com/anm }\end{array}$ & $\begin{array}{l}\text { This article is licensed under the Creative Commons Attribution- } \\
\text { NonCommercial-NoDerivatives } 4.0 \text { International License (CC BY- } \\
\text { NC-ND) (http://www.karger.com/Services/OpenAccessLicense). } \\
\text { Usage and distribution for commercial purposes as well as any dis- } \\
\text { tribution of modified material requires written permission. }\end{array}$ \\
\hline
\end{tabular}

Etienne Macedo

Department of Medicine

University of California San Diego

9500 Gilman Dr, La Jolla, CA 92093 (USA)

E-Mail emmacedo@ucsd.edu 
points were essential for the initiative: defining preventable death from AKI and promoting local recommendations for AKI care considering the health-care infrastructure and socioeconomic conditions [1, 16-19]. Based on previous studies, preventable deaths from AKI are known to occur as a result of 3 different situations [1]: (1) secondary to public health problems such as unclean water, diarrhea, and endemic infections; (2) delayed or lack of recognition, lack of access to laboratory studies, inadequate response, or iatrogenic factors resulting in additional insults to a failing kidney; and (3) lack of dialysis support to treat lifethreatening hyperkalemia, fluid overload, and acidosis [1].

Although knowledge of the epidemiology of AKI has improved immensely since the use of a standardized AKI classification system, few studies have focused on communityacquired AKI in low-resource settings. In the meta-analysis by the 0 by 25 initiative, the main issues regarding the epidemiology of AKI were raised [1]. Information was presented regarding the increasing associated mortality of even mild AKI, the effects of an AKI episode on long-term outcomes, and early detection and treatment of AKI in outpatient and low-resource settings. However, to reduce AKIrelated mortality and morbidity, knowledge of the factors that affect AKI outcomes is a key step in implementing initiatives. The strategies to reduce the burden of AKI need to be based on the identification of patients at risk, implementation of preventive actions, application of diagnostic methods, and timely referral for specialist care [20,21]. Development of educational and training tools for raising awareness and standardizing care of AKI cases is also essential.

As most studies on AKI are derived from developed countries and mainly focus on ICU populations, the 0 by 25 initiative developed 2 projects to assess how AKI contributes to the global burden of healthloss: the Global AKISnapshot (GSN) study, and the Pilot Study. The GSN study was a prospective multinational cross-sectional project that included all patients with AKI who presented to the participating physicians on a given index day in 2014 [2]. The study included 4,018 patients with AKI across 6 continents and 72 countries. The Pilot Study is a prospective cohort of patients with high risk for community-acquired AKI in 3 different countries $[3,4]$. The following paragraphs will comment on the main findings from the 0 by 25 initiative so far.

\section{AKI Meta-Analysis}

A systematic search of the literature was performed, including papers from January 2012 to August 2014. Four hundred and ninety-nine papers that included all AKI def- initions, plus 266 papers based on KDIGO or equivalent AKI definitions, were analyzed [1]. The pooled incidence rate by KDIGO stage in 266 studies $(4,502,158$ subjects) showed an overall rate of $20.9 \%$ of hospital admissions and affected 3,000-5,000 patients per 1 million population per year. Recent studies have described an incidence as high as 15,000 per 1 million population per year. The data from these studies showed that the mortality rates continue to be high in all regions and that there was a continued association of nonrenal recovery following AKI.

Nevertheless, AKI incidence in low-middle income countries (LMICs) is still uncertain as some studies have shown lower levels than in high-income countries (HICs). It is likely that underreporting is the most common reason for the discrepancy when comparing HICs and combined low-incomes plus LMIC. In addition, epidemiological data from LMICs are difficult to interpret as there are nonuniform cohorts and involve heterogeneous methods of reporting as well as wide variations in ability to diagnose and treat AKI [1].

Another factor to consider is the high incidence of AKI in the hospital settings of areas with more resources, in contrast to community hospitals and rural areas, where AKI is often not detected $[8,19,22-26]$. Nonetheless, AKI in this population is often preventable and reversible, affecting young, previously healthy individuals and might be secondary to tropical infectious diseases, animal venoms, the use of herbal medicine, complications of pregnancy including septic abortion, and infectious diarrhea (Table 1).

\section{GBD Study}

The Global Burden of Disease (GBD) Study is an effort of the World Health Organization to quantify leading causes of health loss secondary to illness or injury throughout the world [27]. The GBD Study categorizes causes of health loss by age, sex, and geography for a specific time point. This time-based measure combines years of life lost due to premature mortality (YLL) and years of life lost due to time lived in states of less than full health (DALY). The DALY metric was developed in the original GBD 1990 study to assess the burden of disease consistently across diseases, risk factors, and regions.

As a part of the 0 by 25 initiative, the ISN has collaborated with the Institute of Health Metrics and Evaluation that coordinates the GBD study to include AKI in future GBD reports. Incorporating AKI into the GBD will involve determining the relationship between $\mathrm{AKI}$ as an intermediate event associated with disability or death. It
Macedo/Garcia-Garcia/Mehta/Rocco 
Table 1. Main risk factors for developing AKI

\begin{tabular}{|c|c|c|c|}
\hline \multicolumn{2}{|l|}{ Patient } & \multicolumn{2}{|l|}{ Exposures } \\
\hline Chronic kidney disease & Intravascular volume & Obstetric complications (including & Limited clean water availability \\
\hline Diabetes mellitus & depletion & septic absorption) & Inadequate control of parasites \\
\hline Cancer & Hypotension & Infectious diseases (malaria, & Inadequate control of \\
\hline Chronic gastrointestinal & Use of nephrotoxic & Animal venoms (snakes, bees and & Inadequate health budget \\
\hline disease & agents (antibiotics, & wasps, Loxosceles spiders, Lonomia & Insufficient health care human \\
\hline Demographic factors & iodinated contrast, & caterpillars) & resources \\
\hline Gender & nonsteroidal & Natural medicines & Insufficient health services and \\
\hline Older age & anti-inflammatory drugs, & Natural dyes & hospitals \\
\hline
\end{tabular}

Modified from [1].

AKI, acute kidney injury.

will be possible to follow the leading causes of AKI and the segments of the population most susceptible to AKIrelated health loss. The main goal is to add strength to the concept that a high proportion of cases of AKI in the community setting of low resource areas are preventable; it also attempts to demonstrate that investment toward early recognition can be translated into reduce mortality and improve outcomes.

In order to enable the inclusion of AKI in GBD, the ISN 0 by 25 initiative helped to generate AKI epidemiological data at the population level. The 0 by 25 initiative enabled the AKI Global Snapshot, a prospective observational cohort study, to compare risk factors, etiologies, diagnoses, management, and outcomes of AKI. The study was conducted from September 29, 2014, to December 7, 2014, with over 600 participating centers in over 93 countries [2]. Patients were classified as having community-acquired AKI if they presented with AKI and hospital-acquired AKI if they developed it in the hospital setting. Patients were considered as de novo AKI, AKI on CKD, or AKI with unknown prior kidney history if a baseline creatinine was not known. Countries were classified into HICs, upper-middle-income countries (UMICs), and LMICs according to their 2014 gross national income per person, using thresholds defined by the World Bank Atlas method [28].

Overall, community-acquired AKI was more frequent than hospital-acquired AKI, and the difference was greater in LMIC, where $79 \%$ of AKI cases occurred in the com- munity. Most patients (46\%) were at the ward or stepdown unit when AKI diagnosis occurred, with similar rates across all country categories. Eighty percent of the cases were considered de novo AKI.

Hypotension/shock and dehydration were the more frequent risk factors associated with AKI development. In HIC and UMIC, hypotension/shock was the most prevalent cause, whereas dehydration was the most frequent contributory factor for the development of AKI in LMIC. Most dehydration episodes were associated with inadequate oral intake $(60 \%)$, followed by vomiting (44\%).

Patients with stage 3 AKI were higher in LMICs than in HICs and UMICs (58 vs. 47 and 41\%, respectively). However, more patients in LMICs experienced recovery from AKI than did patients from HICs and UMICs. The large proportion of patients presenting with stage 3 AKI has important implications.

In a separate analysis of children, the main factors associated with AKI in HIC were hypotension (30\%), postsurgical complications (27\%), and dehydration (26\%). In contrast, dehydration was the most common etiologic factor in LMIC (43.5\%) and UMIC (30.6\%) [9].

Mortality rate varied from $11.45 \%$ in patients from LMIC to $13.6 \%$ in patients from UMIC. In pediatric patients, the mortality rate was significantly different (19.6\%) in LMIC compared to 1.2\% in HIC [9]. Mortality in community-acquired AKI was higher in LMIC (11\%) 


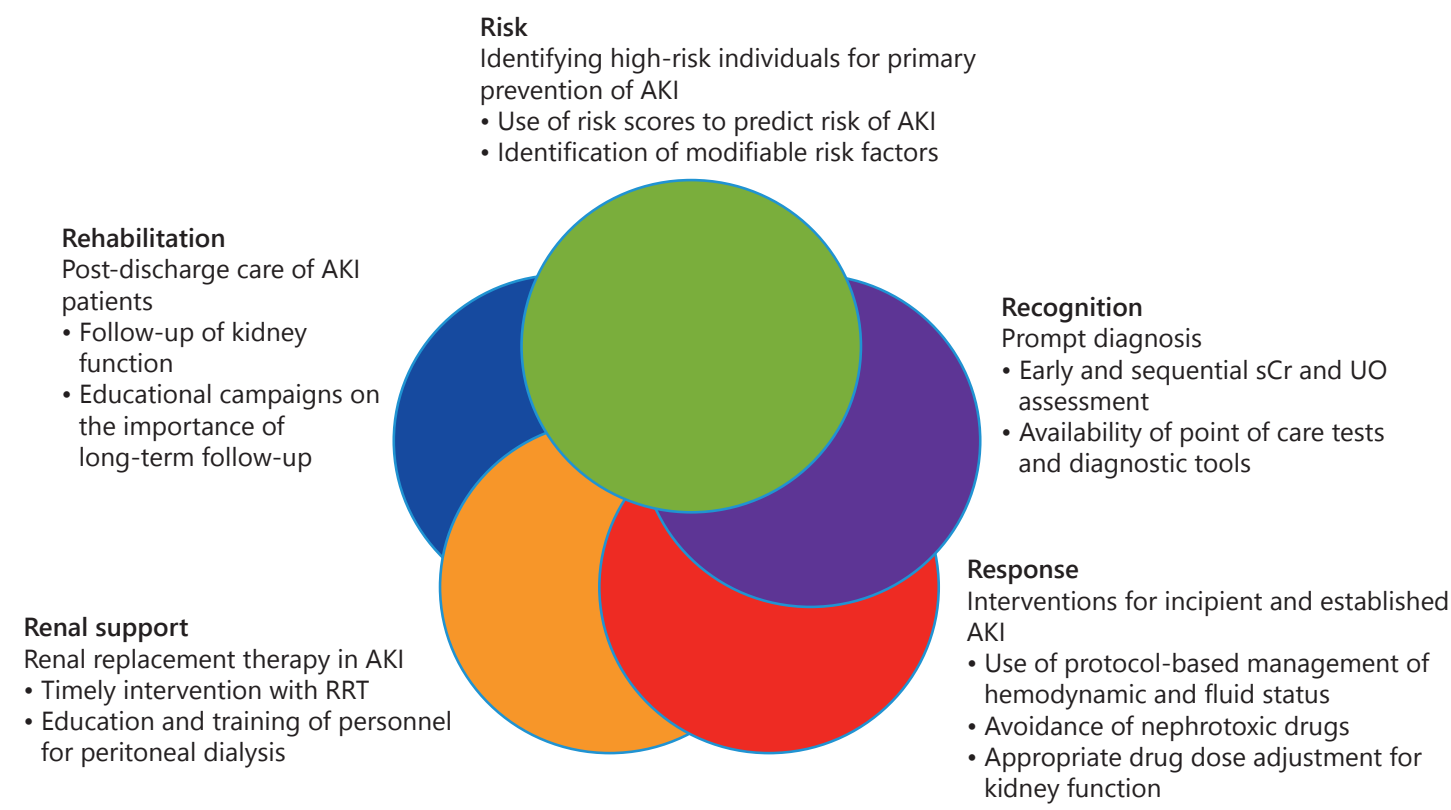

Fig. 1. ISN AKI 0 by 25 key elements for a sustainable infrastructure to support AKI care. sCr, serum creatinine; RRT, renal replacement therapy; UO, urine output; AKI, acute kidney injury. Modified from [1].

vs. $9 \%$ in HIC. In the pediatric population, this difference was even more pronounced, $3 \%$ in HIC and 20\% in LMIC. In LMIC, mortality was higher among ICU patients (21\%) in comparison to HIC (13\%). AKI recovery was more often complete in LMIC (39\%) than in HIC (33\%) or UMIC (28\%). Recovery rates from community vs. acquired AKI were very similar in HIC and UMIC. In LMIC, the recovery occurred in $79 \%$ of patients with community-acquired AKI and in only $20 \%$ of patients with hospitalacquired AKI.

The results of the GSN underline the need to raise awareness of AKI to increase the detection of patients who present with earlier stages of AKI. It also indicates that the main causes of AKI in LMICs are dehydration, infection, and sepsis.

\section{Pilot Study}

In the GSN study [2], we were able to demonstrate that there are significant similarities in the risk factors and causes of AKI worldwide; however, there was an underrepresentation of community-acquired AKI, particularly in rural settings. The primary aim of the 0 by 25 AKI Pilot Feasibility Project was to assess the feasi- bility of implementing an education and training program to optimize care of AKI, based on a protocol-driven approach in rural areas. The Pilot feasibility study was conducted at 3 sites located in Asia (Dharan, Nepal), Africa (Blantyre, Malawi), and Latin America (Cochabamba, Bolivia). Each site comprised a health-care cluster (including 3-4 community health centers, 1 district hospital, 1 regional referral hospital) that provided services to the population around the site area. The study was approved by the Institutional Review Board and the Ethics Committee of University of California San Diego and by the 3 local sites. Patients were screened for signs or symptoms a priori associated with high/ moderate risk of developing AKI. AKI was confirmed within 7 days by a serum creatinine concentration increase or decrease of $0.3 \mathrm{mg} / \mathrm{dL}$, or 1.5 times from the reference value.

The results of the pilot study, soon to be published, will provide an assessment of the current approach to diagnosis and management of AKI in community health centers and will identify barriers to optimize care of these patients. It will demonstrate the effect of simple interventions, including education and provision of point-of-care tests, on the course and outcomes of patients with a high risk of developing AKI. As a part of the project, we devel- 
oped partnerships with the governments of the participating countries to establish the best approaches to decrease preventable deaths from AKI.

\section{Next Steps}

AKI has been associated with high mortality rates; however, it is likely that a significant number of deaths associated with AKI could be avoided. In addition, AKI is now a recognized important risk factor for new-onset $\mathrm{CKD}$, determining acceleration in progression to endstage renal disease, leading to poor quality of life, disability, and long-term costs [29]. The Global Snapshot was the first large, epidemiologic study to map and scale the outcomes associated with AKI around the world, including data from ICU and non-ICU patients. It provided a solid basis to direct efforts for the ambitious goal of zero deaths from AKI by 2025.

The ISN 0 by 25 initiative offers a critical opportunity to help improve education, training, care delivery, and the implementation of diagnostic and intervention studies in AKI. A comprehensive approach for education and training of health-care personnel is fundamental to achieve increased awareness and better care delivery in AKI. Additional key elements include improvement in health care and diagnostic tool availability and provision of acute renal replacement therapy for those in need. The worldwide heterogeneity in the cause, setting, and course of AKI demands an integrative approach. The 0 by 25 initiative proposed the utilization of the $5 \mathrm{R}$ framework: Risk assessment, Recognition, Response, Renal support, and Rehabilitation (Fig. 1) [1, 30].

Furthermore, this initiative is enabling the development of sustainable infrastructure to improve education, training, care delivery, and implementation of diagnostic and intervention studies. It provided evidence suggesting that the majority of AKI cases would be treatable and often reversible, with early identification in high-risk patients and implementation of basic treatment.

\section{Disclosure Statement}

E.M., G.G.-G., and M.R. received travel expenses and registration fee from Danone Research to participate in the 2018 Hydration for Health Scientific Conference. R.L.M. has nothing to disclose.

\section{References}

1 Mehta RL, Cerdá J, Burdmann EA, Tonelli M, García-García G, Jha V, et al. International Society of Nephrology's 0by25 initiative for acute kidney injury (zero preventable deaths by 2025): a human rights case for nephrology. Lancet. 2015 Jun;385(9987):2616-43.

2 Mehta RL, Burdmann EA, Cerdá J, Feehally J, Finkelstein F, García-García G, et al. Recognition and management of acute kidney injury in the International Society of Nephrology 0by25 Global Snapshot: a multinational cross-sectional study. Lancet. 2016 May; 387(10032):2017-25.

3 Macedo E. Use of a serum creatinine point of care test in low resource settings: correlation and agreement with hospital based assessment. In: Sharma S, Hemmila U, Claure-Del Granado R, Cerda J, Burdmann EA, Rocco M, et al., editors. J Am Soc Nephrol. 2017. p. 415.

4 Macedo E. Spectrum of AKI in Patients from Low Income Countries Participating in the ISN0by25 Initiative. In: Sharma KS, editor. J Am Soc Nephrol - Abstract Supplement; October 2017.

5 Mesropian PD, Othersen J, Mason D, Wang J, Asif A, Mathew RO. Community-acquired acute kidney injury: A challenge and opportunity for primary care in kidney health. Nephrology (Carlton). 2016 Sep;21(9):72935.
6 Sawhney S, Fluck N, Fraser SD, Marks A, Prescott GJ, Roderick PJ, et al. KDIGO-based acute kidney injury criteria operate differently in hospitals and the community-findings from a large population cohort. Nephrol Dial Transplant. 2016 Jun;31(6):922-9.

7 Hoste EA, Kellum JA, Selby NM, Zarbock A, Palevsky PM, Bagshaw SM, et al. Global epidemiology and outcomes of acute kidney injury. Nat Rev Nephrol. 2018 Oct;14(10):607-25.

8 Hsu CN, Chen HL, Tain YL. Epidemiology and outcomes of community-acquired and hospital-acquired acute kidney injury in children and adolescents. Pediatr Res. 2018 Mar; 83(3):622-9.

9 Macedo E, Cerdá J, Hingorani S, Hou J, Bagga A, Burdmann EA, et al. Recognition and management of acute kidney injury in children: the ISN 0by25 Global Snapshot study. PLoS One. 2018 May;13(5):e0196586.

10 Coca SG, Yusuf B, Shlipak MG, Garg AX, Parikh CR. Long-term risk of mortality and other adverse outcomes after acute kidney injury: a systematic review and meta-analysis. Am J Kidney Dis. 2009 Jun;53(6):961-73.

11 Daher EF, Silva Junior GB, Santos SQ, R Bezerra CC, Diniz EJ, Lima RS, et al. Differences in community, hospital and intensive care unit-acquired acute kidney injury: observational study in a nephrology service of a de- veloping country. Clin Nephrol. 2012 Dec; 78(6):449-55.

12 Susantitaphong P, Cruz DN, Cerda J, Abulfaraj M, Alqahtani F, Koulouridis I, et al.; Acute Kidney Injury Advisory Group of the American Society of Nephrology. World incidence of AKI: a meta-analysis. Clin J Am Soc Nephrol. 2013 Sep;8(9):1482-93.

13 Chawla LS, Kimmel PL. Acute kidney injury and chronic kidney disease: an integrated clinical syndrome. Kidney Int. 2012 Sep; 82(5):516-24.

14 Coca SG. Long-term outcomes of acute kidney injury. Curr Opin Nephrol Hypertens. 2010 May;19(3):266-72.

15 Coca SG, Singanamala S, Parikh CR. Chronic kidney disease after acute kidney injury: a systematic review and meta-analysis. Kidney Int. 2012 Mar;81(5):442-8.

16 Yang L, Xing G, Wang L, Wu Y, Li S, Xu G, et al.; ISN AKF 0by 25 China Consortiums. Acute kidney injury in China: a cross-sectional survey. Lancet. 2015 Oct;386(10002):1465-71.

17 Feehally J. The ISN 0by25 Global Snapshot Study. Ann Nutr Metab. 2016;68(Suppl 2): 29-31.

18 Perico N, Remuzzi G. Prevention programs for chronic kidney disease in low-income countries. Intern Emerg Med. 2016 Apr; 11(3):385-9. 
19 Wang Y, Wang J, Su T, Qu Z, Zhao M, Yang L, et al.; ISN AKF 0by25 China Consortium. Community-Acquired Acute Kidney Injury: A Nationwide Survey in China. Am J Kidney Dis. 2017 May;69(5):647-57.

20 Chawla LS, Amdur RL, Shaw AD, Faselis C, Palant CE, Kimmel PL. Association between AKI and long-term renal and cardiovascular outcomes in United States veterans. Clin J Am Soc Nephrol. 2014 Mar;9(3):44856.

21 Leung KC, Tonelli M, James MT. Chronic kidney disease following acute kidney injuryrisk and outcomes. Nat Rev Nephrol. 2013 Feb;9(2):77-85.

22 Jha V, Chugh KS. Community-acquired acute kidney injury in Asia. Semin Nephrol. 2008 Jul;28(4):330-47.
23 Emmett L, Tollitt J, McCorkindale S, Sinha S, Poulikakos D. The Evidence of Acute Kidney Injury in the Community and for Primary Care Interventions. Nephron. 2017;136(3): 202-10.

24 Holmes J, Geen J, Phillips B, Williams JD, Phillips AO, Welsh AKI Steering Group. Community acquired acute kidney injury: findings from a large population cohort. QJM. 2017 Nov;110(11):741-6.

25 Inokuchi R, Hara $\mathrm{Y}$, Yasuda $\mathrm{H}$, Itami N, Terada Y, Doi K. Differences in characteristics and outcomes between community- and hospitalacquired acute kidney injury: A systematic review and meta-analysis. Clin Nephrol. 2017 Oct;88(10):167-82.

26 Bernardo EO, Cruz AT, Buffone GJ, Devaraj S, Loftis LL, Arikan AA. Community-acquired Acute Kidney Injury Among Children Seen in the Pediatric Emergency Department. Acad Emerg Med. 2018 Jul;25(7):758-68.
27 Murray CJ, Ezzati M, Flaxman AD, Lim S, Lozano R, Michaud C, et al. GBD 2010: design, definitions, and metrics. Lancet. 2012 Dec; 380(9859):2063-6.

28 World Bank. GNI per capita, Atlas method (current US\$) [cited April 2015]. Available from: https://data.worldbank.org/indicator/ ny.gnp.pcap.cd.

29 Sawhney S, Marks A, Fluck N, Levin A, McLernon D, Prescott G, et al. Post-discharge kidney function is associated with subsequent ten-year renal progression risk among survivors of acute kidney injury. Kidney Int. 2017 Aug;92(2):440-52.

30 Lewington AJ, Cerdá J, Mehta RL. Raising awareness of acute kidney injury: a global perspective of a silent killer. Kidney Int. 2013 Sep; 84(3):457-67. 